\title{
New approaches for tissue engineering: three dimensional cell patterning using inkjet technology
}

\author{
Chizuka Henmi ${ }^{1)}$, Makoto Nakamura ${ }^{1,2, *)}$, Yuichi Nishiyama1), \\ Kumiko Yamaguchi²), Shuichi Mochizuki ${ }^{3)}$, Koki Takiura' ${ }^{4)}$, \\ and Hidemoto Nakagawa1) \\ ${ }^{1)}$ Kanagawa Academy of Science and Technology, Kawasaki, Japan \\ 2)Tokyo Medical and Dental University, Tokyo, Japan \\ ${ }^{3)}$ Osaka Institute of Technology, Osaka, Japan \\ 4)Yamagata University, Yonezawa, Japan
}

\begin{abstract}
Tissues and organs are composed of different types of cells and proteins, which interact and express their respective functions based on their three dimensional (3D) structures. Thus, the position and arrangement of those components including cells are critical for tissue engineering, and it is necessary to develop effective technologies for the arrangement of those materials in 3D space. Inkjet printing technology is able to print with small droplets at high resolution that are nearly equal to microscopic tissue structures. We have attempted to use this technology to print with biological cells instead of standard ink and demonstrated that living cells could be safely ejected with the inkjet procedure. Herein, we briefly introduce and review this new approach of inkjet-based cell patterning, which has good potential as an effective tool for tissue engineering.

Rec.2/20/2007, Acc.11/19/2007, pp36-40

\footnotetext{
* Correspondence should be addressed to:

Makoto Nakamura, Kanagawa Academy of Science and Technology, Think-E Building, 1-23 Minamiwatarida, Kawasakiku, Kawasaki, 210-0855, Japan. Phone: +81-44-355-7483, Fax:+81-44-355-7484, e-mail: maknaka.ao@tmd.ac.jp
}

Key words inkjet printing, cell patterning, tissue engineering

\section{Introduction}

Tissue engineering is one of the most promising and reasonable approaches to repair and replace the organ and tissue functions that has been lost or damaged due to several diseases, injuries, aging and congenital deformities. As it has a potential of radical cure for irreversible organ failure, many challenges have been addressed to engineer biological tissues and organs.

Tissues and organs are histologically composed of multiple types of cells and extracellular matrix proteins with three-dimensional (3D) structures. Those components interact with each other and the cells can play their respective roles, and as a result, tissues can express their respective functions. Therefore, the spatial position and the arrangement of those components including cells are very important in bio-functional tissues. For manufacturing such functional tissues by engineering approach, it is necessary to arrange such components at the suitable positions with 
corresponding resolutions. However, there are no established methods for arrangement of several different materials including individual cells in 3D space at high resolution.

Recently, various 3D manufacturing technologies have been applied in tissue engineering field. Especially, rapid manufacturing techniques, such as solid free forming, powder based inkjet 3D-Z-printing, stereo lithography and dispenser, etc. have been applied to manufacturing of 3D scaffolds towards more sophisticated tissue engineering ${ }^{1-5)}$. Those techniques are indeed effective in advancement of scaffold based tissue engineering. However, some serious obstacles are still remaining. Distribution and arrangement of the seeded cells cannot be controlled in the scaffolds at all, especially in the case of different multi kinds of cells. In addition, the local growth factor concentration in the scaffold cannot be controlled. Those essential factors are still uncontrollable by engineering technology, even if morphologically sophisticated scaffolds are used.

We speculated it is because scaffold based tissue engineering is based on the method by homogeneous cell seeding and homogeneous growth factor administration onto overall the scaffolds. Then, in order to overcome those limitations, it is needed to develop some effective technologies to manufacture $3 \mathrm{D}$ structures in which living cells, biological components and bio-functional materials are spatially arranged. In addition, considering to the histological features of tissues, the required technologies should enable to manufacture microscopic architectures and heterogeneous structures with multiple types of cells and biological materials.

Inkjet printers are able to eject microscopic droplets of ink on demand and provide high resolution printing with photographic quality. And inkjet can deal with multiple different color inks to print color pictures. We considered that such abilities of inkjet printing technology must be effective in cell handling and cell positioning with micro-scaled resolution.

In this paper, we would like to introduce our researches on tissue engineering applying inkjet technology, termed "inkjet 3D bioprinting".

\section{Advantages of inkjet technique for 3D bioprinting}

Previously, we demonstrated the advantages of inkjet printing for tissue engineering ${ }^{6}$. The size of single dot printed by a commercial inkjet printer was 20 to $30 \mu \mathrm{m}$ in diameter, which is close to the size of 1 or 2 living cells. The ability to print with several different color inks enable to position different biomaterials and cells onto predetermined positions. And, inkjet printers can eject more than 1000 drops/second from each nozzle, which is advantageous for high speed fabrication of biological living materials. In addition, since inkjet printers are used as an output device of computers, this close relationship with computer technology is advantageous in computer-assisted tissue engineering that is tissue engineering based on computer assisted designing (CAD) and manufacturing (CAM) technologies. Furthermore, we examined whether inkjet can be used for cell printing and confirmed that the printing procedure by inkjet does not affect any significant damages to cells and that inkjet is an available technique for living cell arrangement. And the printer head does not come into contact with the fabricated substrate in inkjet printing, which is important to maintain soft structures during fabrication process. This non-contact printing ability also contributes to develop gelation technique and 3D fabrication technique by inkjet, which is described latter. Those all characteristics and performance of inkjet technology meet the requirements of reasonable manufacturing technology for 3D biological structures in which multiple cells and biological materials are spatially arranged.

\section{Inkjet-based cell patterning by gelling of inkjet droplets}

We recognized the advantages and the potentials of inkjet in tissue manufacturing, and examined cell printing. But, through those experiences, we found simultaneously some problems to solve. Inkjet droplets are so small that they dried very immediately after ejected. On the contrary, when the substrate for printing is wet, drawing pattern is very difficult due to blotting, mixing and diffusion of ink. Furthermore, usual inkjet is for 2D printing, although we need to manufacture 3D structures.

Considering the solution of those problems, we developed a gel formation technique, applying non-contact printing ability of inkjet. Gel precursor was ejected into the substrate of gel reactant by inkjet. Then, inkjet droplets formed hydrogel beads at the landing position. We mainly used sodium alginate as a gel precursor and $\mathrm{CaCl}_{2}$ as a gel reactant, since sodium alginate gels at once by the contact with calcium ion and alginate hydrogel is known as a biocompatible material used in tissue transplantation, such as pancreatic islets transplantation ${ }^{7)}$. Using cell suspension with sodium alginate solution, we succeeded to embed individual living cells in alginate hydrogel beads. This inkjet gelation technique enabled to make 2D and 3D patterning of hydrogel structure containing living cells, without drying, without blotting even in watery medium. 


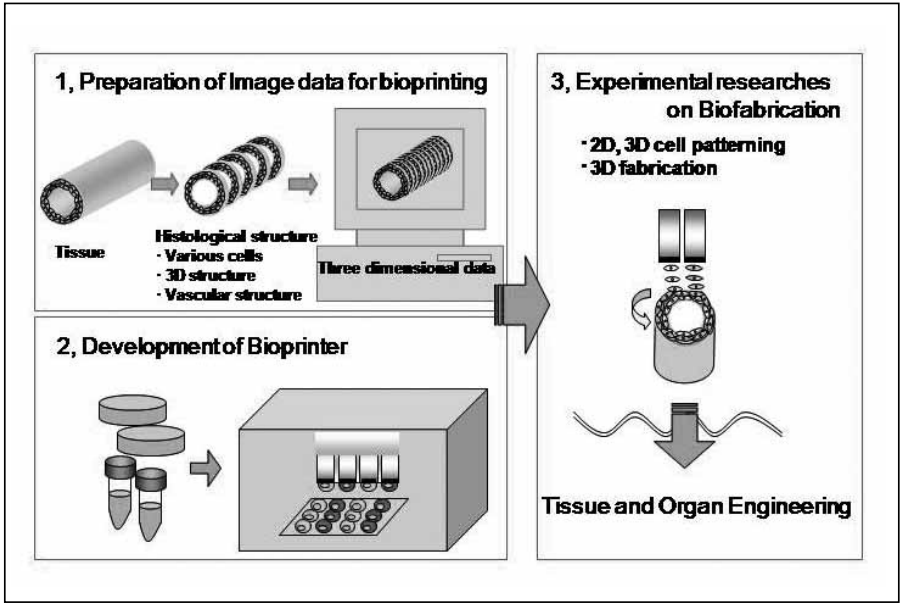

Fig.1 Concept of the development of Bioprinting

\section{Outline of the approach of inkjet-based 3D bioprinting towards tissue and organ engineering}

An outline of the approach of inkjet-based tissue engineering, termed 3D bioprinting, is shown in Figure 1.

1) Designing of tissue structures (Bio-CAD): One of the advantages of 3D bioprinting approach is that designed tissue can be manufactured. We prepared some image data for printing biological tissue structures. We obtained serial sections from some tissues and organs at $5 \mu \mathrm{m}$ in thickness and then, microscopic image data of them were obtained. Those image data were digitally stacked using 3D reconstruction software. 3D digital data of biological tissues and organs were obtained. They will be useful as one of the $\mathrm{CAD}$ data for $\mathrm{CAD} / \mathrm{CAM}$ based tissue engineering.

2) Development of 3D Bioprinter: a bio-CAM machine which realizes 3D manufacturing using living cells and biological materials was unprecedented. Then, we have developed an original 3D Bioprinter by ourselves. The details about the prototype are described latter.

3) Experimental researches on biofabrication: Towards manufacturing some functional biological tissues, we started to examine what structures can be designed and fabricated by 3D bioprinter and how the fabricated 3D structures containing living cells should be cultured to develop them to functional biological tissues. Although many, many efforts and developments are needed to make matured functional tissues and organs available for transplantation, such challenging researches should be required.

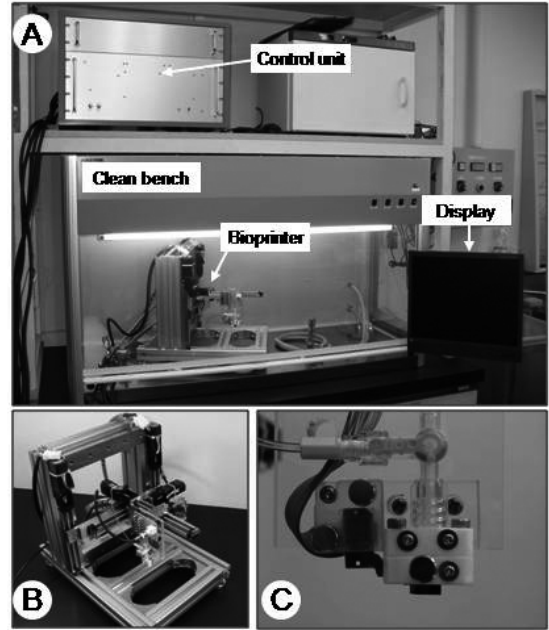

"Bioprinter" Specifications

\begin{tabular}{ll}
\hline \hline Technology & Inkjet \\
Nozze & SEA-Jet ${ }^{\mathrm{IM}}$ (EPSON), etc. \\
Maximum operating frequency & $3 \mathrm{kHz}$ \\
Positioning resolution & $0.2 \mu \mathrm{m}$ \\
Repeated positioning accuracy & $\pm 4 \mu \mathrm{m}$ \\
Maximum speed & $130 \mathrm{~mm} / \mathrm{s}$ \\
Maximum operating distance $(\mathrm{X}, \mathrm{Y}, \mathrm{Z})$ & $100 \times 100 \times 100(\mathrm{~mm})$ \\
Dimensions $(\mathrm{W} \times \mathrm{D} \times \mathrm{H})$ & $400 \times 300 \times 380(\mathrm{~mm})$ \\
\hline
\end{tabular}

Fig.2 An original inkjet system and bioprinter of specifications

A: Our original inkjet system.

B: Overview of bioprinter.

$\mathrm{C}$ : Inkjet head. The arm with the inkjet head moves freely in the $x, y$, and $z$ directions, with the action regulated by use of computer software.

\section{Development of original inkjet 3D bioprinter system}

Although inkjet printers for printing images are commercially sold, the primary use is not to apply in tissue engineering nor in 3D fabrication, but to print only 2D photographs and graphics with specially formulated inks. Then, we have developed unprecedented 3D-bioprinter by ourselves, specialized for 3D fabrication with biological materials including living cells. Shown in Figure 2A and B are the first prototype. The arm with the inkjet head can be freely moved in the $\mathrm{x}, \mathrm{y}$, and $\mathrm{z}$ directions in 3D space. The system was designed to be used inside a clean bench for cell cultures that require a clean environment. We utilized some available inkjet heads selected from already established inkjet heads, such as a SEAJet ${ }^{\mathrm{TM}}$ inkjet head system (Seiko Epson Corp., Suwa, Japan), which is an electrostatically-actuated 


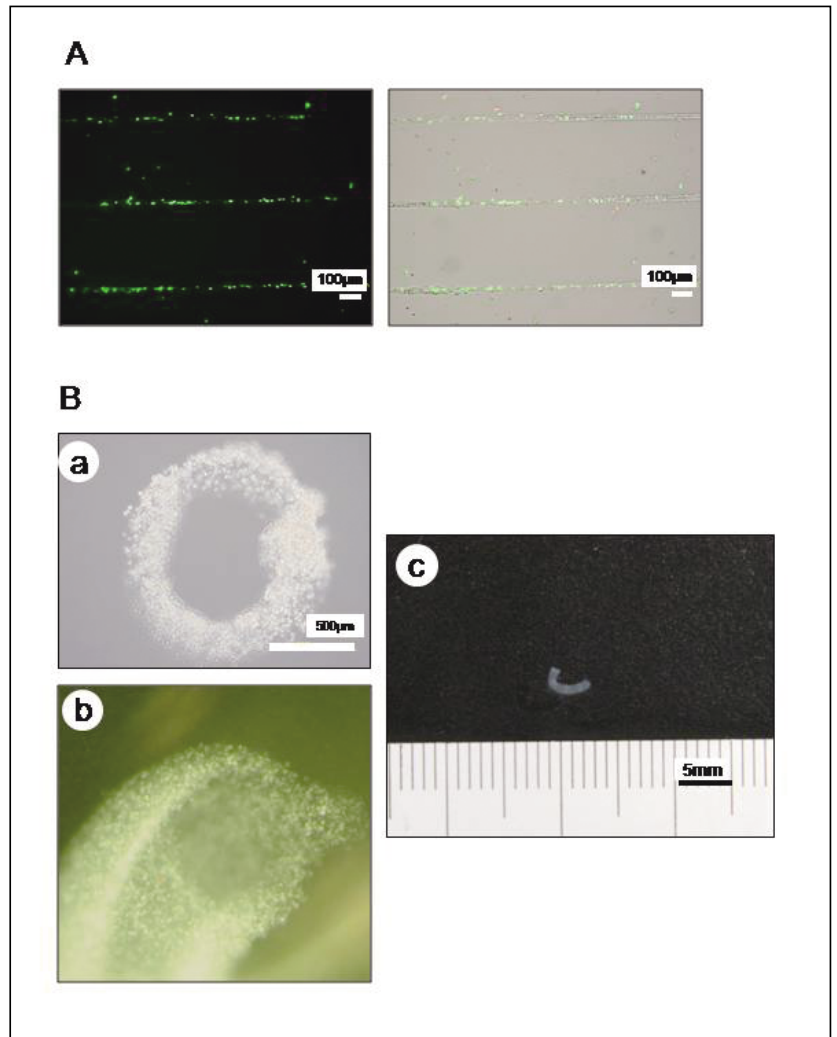

Fig.3 Inkjet-based cell patterning

A: 2D patterning. Fluorescent green stained cells were suspended in medium with $0.8 \%$ sodium alginate solution, after which the mixture was patterned in a linear manner onto solution that included $\mathrm{CaCl} 2$. The alginate gel containing cells arranged in a programmed line pattern by the bioprinter.

B: 3D patterning. Cells suspended in sodium alginate were formed in a circle pattern (a). The tubular structure was fabricated by laminated printing of 2D circular patterns (b). The length of the 3D tubular structure was approximately $5 \mathrm{~mm}$, with a diameter of $1 \mathrm{~mm}$ (c).

type of inkjet head (Fig.2C). The structure and mechanism of the SEAJetTM system has been described previously ${ }^{6}$. Except for the inkjet head system, both the hardware and software of $3 \mathrm{D}$ bioprinter were designed and developed by our research team. Specification for the bioprinter is shown in Fig. 2.

\section{D cell patterning using 3D bioprinter}

Using 3D bioprinter, we tried $2 \mathrm{D}$ cell patterning. We could successfully print living cells with linear patterns without blotting even in a $\mathrm{CaCl}_{2}$ solution (Fig.3A). In those patterns, living cells were embedded in alginate hydrogel fibers of $40 \mu \mathrm{m}$ in diameter. Before developing the gel formation technique, such clear 2D patterns of living cells could not be made by inkjet, because of drying on dried substances, or blotting on a wet substrate.

\section{Fabrication of 3D tubular structures}

Using 3D bioprinter, fabrication of 3D structure containing living cells was challenged. Cell suspension with sodium alginate was overprinted 30 times in a circle pattern onto $\mathrm{CaCl}_{2}$ solution, and a 3D circular or ring structure containing living cells which had some thickness was successfully created (Fig.3Ba). Then, a 3D tubular structure with much more thickness was also fabricated by laminated printing of a circle pattern. The diameter of tubular structure was approximately $1 \mathrm{~mm}$ (Fig.3Bb,c). The length of tube was dependent on the fabricating time. Approximately $5 \mathrm{~mm}$ length tube was produced in one minute, and a longer gel tube which length was more than $1.5 \mathrm{~cm}$ was also obtained using our 3D bioprinter. In addition, we could observe many of the embedded cells were survived in the gel tube.

\section{Perspective}

Recently, inkjet technology have been applied in biological research fields and the technology used to print various biomaterials, including nucleic acid ${ }^{8)}$ and growth factors ${ }^{9}{ }^{90}$ for biochips. Furthermore, biological cells have been used instead of printing ink ${ }^{6,11,12)}$. However, inkjet-based cell patterning technique had not yet been established. In the present study, we demonstrated viable inkjet-based cell patterning for both 2D and 3D structures by gelling of the cell containing droplets and use of laminated printing, indicating that inkjet technology is an effective tool for 2D and 3D tissue engineering.

To create a suitable microenvironment for cells, it is necessary to arrange not only single type of cells but also multi types of cells and biomaterials, such as extracellular matrices and growth factors, around individual cells. Since an inkjet system is proficient at color printing with different materials, tissue fabrication with multiple materials should also be possible. Recently, we have already developed next version of 3D bioprinter for printing with multiple biological cells. By using the 3D bioprinter, we successfully fabricated a dual layered-tube (inner layer: endothelial cells, outer layer: smooth muscle cells), and it was introduced as a topic in material science area ${ }^{13)}$. In addition, it has been reported that a gradient pattern of growth factors could be formed using inkjet printing on 2D fibrin film, and cell proliferation was enhanced within the growth factor patterned region ${ }^{9,10)}$. This indicates that inkjet bioprinting technology has a potential to control cellular functions and tissue growth by controlling the concentration gradients of growth factors in the fabricated struc- 
tures.

In this study, we used only alginate hydrogel for 3D fabrication. However, we have considered it is necessary to arrange a combination of different types of hydrogels, because of a controlling of cellular attachment and differentiation. We have already started to develop $3 \mathrm{D}$ fabrication method using modified alginate hydrogel and fibrin gel.

In the future, we believe that inkjet 3D biofabrication technology will develop more sophisticated 3D biofabrication, in which 3D environment of cells that is the spatial interactions between cell-cell, cell-materials and cell-growth factors, are all designed and controlled by engineering. Although many challenging researches are needed, inkjet 3D biofabrication has a good potential to provide sophisticated 3D tissue structures, and will contribute to the great progress in tissue engineering and life science studies.

\section{Acknowledgments}

We thank Seiko-EPSON Corp. for their support in regard to the inkjet head and Dr. K. Suyama for the expert technical assistance. This work was supported by Kanagawa Academy of Science and Technology, Japan, and Grants-in-aid for Scientific Research (\#17300146, \#18760518 and $\# 18880042$ ) from Japan Society for the Promotion of Science.

\section{References}

1) Yang S, Leong KF, Du Z, Chua CK: The design of scaffolds for use in tissue engineering. Part II. Rapid prototyping techniques, 8: 1-11, 2002.

2) Landers R, Hubner U, Schmelzeisen R, Mulhaupt R: Rapid prototyping of scaffolds derived from thermoreversible hydrogels and tailored for applications in tissue engineering. Biomaterials, 23: 4437-4447, 2002.

3) Griffith LG, Naughton G: Tissue engineering--current chal- lenges and expanding opportunities. Science, 295: 10091014, 2002.

4) Wilson WC, Boland T: Cell and organ printing 1: protein and cell printers. Anat Rec, 272: 491-496, 2003.

5) Sun W, Lal P: Recent development on computer aided tissue engineering-a review. Comput Meth Prog Biomed, 67: 85-103, 2002.

6) Nakamura M, Kobayashi A, Takagi F, Watanabe A, Hiruma Y, Ohuchi K, Iwasaki Y, Horie M, Morita I, Takatani S: Biocompatible inkjet printing technique for designed seeding of individual living cells. Tissue Eng, 11: 1658-1666, 2005.

7) Lim F, Sun AM: Microencapsulated islets as bioartificial endocrine pancreas. Science, 210: 908-910, 1980.

8) Goldmann T, Gonzalez JS: DNA-printing: utilization of a standard inkjet printer for the transfer of nucleic acids to solid supports. J Biochem Biophys Methods, 42: 105-110, 2000.

9) Campbell PG, Miller ED, Fisher GW, Walker LM, Weiss LE: Engineered spatial patterns of FGF-2 immobilized on fibrin direct cell organization. Biomaterials, 25: 6762-6770, 2005.

10) Miller ED, Fisher GW, Weiss LE, Walker LM, Campbell PG: Dose-dependent cell growth in response to concentration modulated patterns of FGF-2 printed on fibrin. Biomaterials, 27: 2213-2221, 2006.

11) $\mathrm{Xu} \mathrm{T}$, Jin J, Gregory $\mathrm{C}$, Hickman JJ, Boland T: Inkjet printing of viable mammalian cells. Biomaterials, 26:93-99, 2005.

12) Saunders RE, Gough JE, Derby B: Delivery of human fibroblast cells by piezoelectric drop-on-demand inkjet printing. Biomaterials, 29: 193-203, 2008.

13) Calvert P: Printing Cells. Science, 318: 208-209, 2007. 